\title{
Perioperative risk factors for prolonged mechanical ventilation after liver transplantation due to acute liver failure
}

\author{
Serin Lee, Hyun Sik Jung, Jong Ho Choi, Jaemin Lee, Sang Hyun Hong, Sung Hyun Lee, and \\ Chul-Soo Park
}

Department of Anesthesiology and Pain Medicine, Seoul St. Mary's Hospital, College of Medicine, The Catholic University of Korea, Seoul, Korea

Background: Acute liver failure (ALF) is a rapidly progressing and fatal disease for which liver transplantation (LT) is the only treatment. Posttransplant mechanical ventilation tends to be more prolonged in patients with ALF than in other LT patients. The present study examined the clinical effects of prolonged posttransplant mechanical ventilation (PMV), and identified risk factors for PMV following LT for ALF.

Methods: We reviewed data of patients undergoing LT for ALF between January 2005 and June 2011. After grouping patients according to administration of PMV ( $\geq 24 \mathrm{~h}$ ), donor and recipient perioperative variables were compared between the groups with and without PMV. Potentially significant factors $(\mathrm{P}<0.1)$ from the univariate intergroup comparison were entered into a multivariate logistic regression to establish a predictive model for PMV.

Results: Twenty-four (25.3\%) of 95 patients with ALF who received PMV had a higher mortality rate (29.2\% vs $11.3 \%$, $\mathrm{P}=0.038)$ and longer intensive care unit stay $(12.9 \pm 10.4$ vs $7.1 \pm 2.7$ days, $\mathrm{P}=0.012)$ than patients without PMV. The intergroup comparisons revealed worse preoperative hepatic conditions, more supportive therapy, and more intraoperative fluctuations in vital signs and less urine output in the with-compared with the without-PMV group. The multivariate analysis revealed that preoperative hepatic encephalopathy ( $\geq$ grade III), intraoperative blood pressure fluctuation, and oliguria $(<0.5 \mathrm{ml} / \mathrm{kg} / \mathrm{h})$ were independent risk factors for PMV.

Conclusions: PMV was associated with deleterious outcomes. Besides care for known risk factors including hepatic encephalopathy, meticulous attention to managing intraoperative hemodynamic circulatory status is required to avoid PMV and improve the posttransplant prognosis in ALF patients. (Korean J Anesthesiol 2013; 65: 228-236)

Key Words: Acute liver failure, Liver transplantation, Mechanical ventilation, Prediction.

Received: January 28, 2013. Revised: 1st, March 8, 2013; 2nd, March 26, 2013. Accepted: April 2, 2013.

Corresponding author: Chul-Soo Park, M.D., Ph.D., Department of Anesthesiology and Pain Medicine, Seoul St. Mary's Hospital, 505, Banpodong, Seocho-gu, Seoul 137-040, Korea. Tel: 82-2-2258-2236, Fax: 82-2-537-1951, E-mail: p6c8s17@catholic.ac.kr

(c) This is an open-access article distributed under the terms of the Creative Commons Attribution Non-Commercial License (http:// creativecommons.org/licenses/by-nc/3.0/), which permits unrestricted non-commercial use, distribution, and reproduction in any medium, provided the original work is properly cited. 


\section{Introduction}

Acute liver failure (ALF) is a fatal disease characterized by rapidly developing hepatocellular dysfunction, severe coagulopathy, and change in mental status without pre-existing liver disease or chronic liver disease [1]. The spontaneous recovery rate for ALF is low; thus, rapid symptomatic deterioration commonly leads to coma and death. The ALF mortality rate is 50-90\% despite medical treatment, and liver transplantation (LT) is the only available treatment [2].

In contrast to chronic liver disease, the physical condition of patients with ALF can be easily and rapidly aggravated. The interval between the development of the initial jaundice or coagulopathy to hepatic encephalopathy is generally short, and multiple organ failure including renal or cardiac dysfunction, and death may follow unless an effective treatment is undertaken. Thus, timely transplantation is critical for the survival of patients with ALF, and emergency LT is more common than an elective surgery. Hepatic encephalopathy in patients with ALF undergoing emergency LT is conspicuous and severe compared with that in LTs for other end-stage liver diseases. Delirium or convulsion frequently occurs, and intracranial hypertension from encephalopathy accounts for $20-25 \%$ of deaths from ALF [3]. Pathophysiologically, ALF accompanies structural changes in the brain including cerebral edema, which occurs in $70-80 \%$ of patients who have severe encephalopathy ( $\geq$ grade 3 ). These changes do not usually recover during the immediate posttransplant period, and patients with ALF frequently require intubation for airway protection from aspiration or mechanical ventilation support in the posttransplant period [4].

Postoperative prolonged intubation is associated with aggravation of the original disease, high incidence of infectious complications, long stay in the intensive care unit, and a high mortality rate in different types of surgeries [5]. A growing body of evidence indicates that mechanical ventilation has a detrimental effect on blood flow to the transplanted graft in post-transplant patients [6]. Accordingly, several transplant centers strive for early posttransplant extubation in elective LT patients for optimal results [7].

Posttransplant ventilation has been widely studied in elective LT; however, few investigators have examined prolonged posttransplant mechanical ventilation (PMV) after LT in patients with ALF. Although LT markedly improves the ALF prognosis, the persistently high mortality rate in patients with ALF compared with elective LT is a matter of concern for many clinicians. Thus, given the profound effect of PMV on prognosis, PMV following LT for ALF is worthy of further study.

The primary goal of the present study was to determine whether PMV following LT for ALF affected prognosis. Further- more, we established a predictive model for PMV after LT for ALF to improve the perioperative management.

\section{Materials and Methods}

Patients (age $\geq 18$ years) who received LT for ALF at our hospital between January 2005 and June 2011 were eligible for the present study. Donor data and recipient preoperative, intraoperative, and postoperative data were collected retrospectively. The Institutional Review Board of our hospital approved the study protocol and patient data collection. The electronic medical recording system and patient charts were used for data collection. ALF was defined as severe and sudden liver dysfunction that induced encephalopathy and coagulopathy (international normalized ratio of prothrombin time $\geq 1.5$ ) within 26 weeks of symptom appearance in patients without pre-existing liver cirrhosis [8].

LTs were performed using widely accepted surgical procedures. All patients received ABO-compatible grafts from living (right hepatic lobes) or deceased donors. In each case, portal vein anastomosis was followed by hepatic artery anastomosis and bile duct reconstruction. A portacaval shunt was used when the difference between the portal venous pressure and central venous pressure was more than $5 \mathrm{mmHg}$ after clamping the portal vein. Balanced anesthesia was performed using isoflurane, vecuronium (or atracurium), and opioids such as fentanyl or remifentanil under a 40-50\% oxygen/air mixture. Mechanical ventilation was controlled in the $\mathrm{PaCO}_{2}$ range of $30-35 \mathrm{mmHg}$ using a tidal volume of $8^{-}-10 \mathrm{ml} / \mathrm{kg}$ and the rate of 10-14 breaths/min. After the anesthetic induction, a SwanGanz catheter was placed through the right internal jugular vein for hemodynamic monitoring and cardiac output measurement, and the radial artery was cannulated using a 22 -guage angiocatheter for blood sampling and continuous monitoring of blood pressure. Intraoperative patient management was guided by the LT protocol of our hospital. Packed red blood cells were administered to maintain hematocrit between 25 and $30 \%$. Fresh frozen plasma, cryoprecipitate, and platelets were replaced to improve intraoperative coagulopathy under thromboelastography guidance. Vasopressors were administered when dangerous hemodynamic instability was indicated by invasive hemodynamic monitors. Furosemide or mannitol was administered with fluid loading when a patient had a urine output less than $0.5 \mathrm{ml} / \mathrm{kg} / \mathrm{h}$. Total change in serum sodium level was minimized for preoperative hyponatremic patients using $0.45 \%$ normal saline. Calcium chloride was administered when serum calcium levels dropped to less than $80 \%$ of the normal lower limit. Sodium bicarbonate was indicated when the serum $\mathrm{pH}$ was lower than 7.25 under adequate minute ventilation and vital signs were unstable. Intracranial pressure 
was not measured because of the risk of bleeding or infection.

Posttransplant extubation was performed according to previously published criteria when: respiratory patterns were stable during a spontaneous breathing trial (respiratory rate $\leq$ $30-35$ breaths/min, tidal volume $\geq 4-6 \mathrm{ml} / \mathrm{kg}$, respiratory rate/ tidal volume ratio $60-105 / \mathrm{L})$, gas exchange was adequate $\left(\mathrm{PaO}_{2}\right.$ $\geq 60 \mathrm{mmHg}$ under $\mathrm{FiO}_{2} \leq 0.4$, PEEP $\leq 5-10 \mathrm{cmH}_{2} \mathrm{O}, \mathrm{PaO}_{2} / \mathrm{FiO}_{2}$ $\geq 150-300$ ), hemodynamic stability (heart rate $\leq 140$ beats/ min, stable blood pressure, no or minimal vasopressors), afebrile (temperature $\leq 38^{\circ} \mathrm{C}$ ), no significant respiratory acidosis, adequate mentation, stable metabolic status (acceptable electrolyte), and subjective comfort [9]. If a patient was hemodynamically unstable or had preoperative encephalopathy, mechanical ventilation or intubation was sustained until vital signs were restored within $10 \%$ of the normal range, or consciousness was recovered to more than light drowsy. The end-point of the study was PMV following LT. PMV was defined according to the literature as mechanical ventilation for more than $24 \mathrm{~h}$ after surgery [10], and patients were categorized as having received (with) or not received (without) PMV.

Preoperative variables included age, body mass index, gender, cause of ALF, Child-Pugh-Turcotte class, model for end-stage liver disease (MELD) score, history of diabetes, hypertension or heart disease, hepatic encephalopathy grade, invasive instrumentation (such as intubation), artificial liver or continuous renal replacement therapy, and laboratory findings. Furthermore, donor data such as age, fatty change of the liver graft, and graftrecipient weight ratio were investigated. Intraoperative variables included surgical time, fluctuations in mean blood pressure (MBP) measured via an arterial line and heart rate in 5-min intervals (defined by the standard deviation; square root of the value calculated by squaring the difference between the mean and individual MBP or heart rate values, summing those numbers, and dividing the sum by the total number of vital signs checked), pulmonary arterial pressure, central venous pressure, hourly urine output, oxygen indices $\left(\mathrm{PaO}_{2} / \mathrm{FiO}_{2}\right)$,

Table 1. Comparison of Preoperative Recipient and Donor Characteristics

\begin{tabular}{|c|c|c|c|}
\hline & \multicolumn{2}{|c|}{ Prolonged posttransplant mechanical ventilation ( $\geq 24 \mathrm{~h})$} & \multirow{2}{*}{$\mathrm{P}$} \\
\hline & No $(n=71)$ & Yes $(n=24)$ & \\
\hline \multicolumn{4}{|l|}{ Recipient preoperative } \\
\hline Age $(y r)$ & $47.7 \pm 11.3$ & $43.1 \pm 13.9$ & NS \\
\hline Body mass index $\left(\mathrm{kg} / \mathrm{m}^{2}\right)$ & $23.7 \pm 3.2$ & $25.0 \pm 2.8$ & NS \\
\hline Gender $(\mathrm{M} / \mathrm{F})$ & $43(60.6 \%) / 28(39.4 \%)$ & $16(66.7 \%) / 8(33.3 \%)$ & NS \\
\hline Cause of acute liver failure (viral/non-viral) & $54(76.1 \%) / 17(23.9 \%)$ & $14(58.3 \%) / 10(41.7 \%)$ & 0.096 \\
\hline Child-Pugh-Turcotte class C & $54(76.1 \%)$ & $21(87.5 \%)$ & NS \\
\hline MELD score (pts) & $25.8 \pm 8.6$ & $33.5 \pm 7.4$ & $<0.001$ \\
\hline Diabetes mellitus & $14(19.7 \%)$ & $4(16.7 \%)$ & NS \\
\hline Hypertension & $12(16.9 \%)$ & $1(4.2 \%)$ & NS \\
\hline Heart diseases & $8(11.3 \%)$ & $2(8.3 \%)$ & NS \\
\hline Severe hepatic encephalopathy (grade III, IV) & $6(8.5 \%)$ & $8(33.3 \%)$ & 0.003 \\
\hline Intubation & $3(4.2 \%)$ & $5(20.8 \%)$ & 0.023 \\
\hline Artificial liver & $6(8.5 \%)$ & $2(8.3 \%)$ & NS \\
\hline CRRT & $10(14.1 \%)$ & $9(37.5 \%)$ & 0.013 \\
\hline \multicolumn{4}{|l|}{ Laboratory finding } \\
\hline Hemoglobin (g/dl) & $10.6(9.1-11.8)$ & $8.6(8.7-9.5)$ & NS \\
\hline Platelet $(/ \mu \mathrm{l})$ & $70000(43000-107000)$ & $76500(49000-111750)$ & NS \\
\hline Neutrophil/lymphocyte ratio & $6.44(2.52-10.56)$ & $6.39(4.31-11.41)$ & NS \\
\hline SGPT (U/L) & $82.0(49.0-204.0)$ & $149.0(76.0-1162.8)$ & 0.058 \\
\hline Creatinine (mg/dl) & $0.92(0.73-1.42)$ & $1.04(0.74-1.91)$ & NS \\
\hline Total bilirubin (mg/dl) & $10.68(3.67-23.64)$ & $20.95(7.78-34.78)$ & 0.025 \\
\hline INR & $2.21 \pm 0.81$ & $2.76 \pm 0.99$ & 0.007 \\
\hline Ammonia $(\mu \mathrm{g} / \mathrm{dl})$ & $177.7 \pm 87.1$ & $185.0 \pm 92.3$ & NS \\
\hline $\mathrm{Na}^{+}(\mathrm{mEq} / \mathrm{L})$ & $138.2 \pm 7.9$ & $140.6 \pm 6.9$ & NS \\
\hline $\mathrm{K}^{+}(\mathrm{mEq} / \mathrm{L})$ & $3.84 \pm 0.66$ & $3.66 \pm 0.77$ & NS \\
\hline $\mathrm{pH}$ & $7.424 \pm 0.095$ & $7.409 \pm 0.106$ & NS \\
\hline \multicolumn{4}{|l|}{ Donor related } \\
\hline Age (yr) & $33.5 \pm 10.8$ & $36.1 \pm 13.4$ & NS \\
\hline Living/deceased donor & $60(84.5 \%) / 11(15.5 \%)$ & $19(79.2 \%) / 5(20.8 \%)$ & NS \\
\hline Fatty change by liver biopsy (\%) & $5.0(2.0-10.0)$ & $4.0(1.0-6.25)$ & NS \\
\hline GRWR (\%) & $1.24 \pm 0.65$ & $1.23 \pm 0.56$ & NS \\
\hline
\end{tabular}

Data are shown as mean \pm SD or median (interquartile range) and number (\%). MELD: model for end-stage liver disease, CRRT: continuous renal replacement therapy, SGPT: serum glutamic pyruvic transaminase, INR: international normalized ratio of prothrombin time, GRWR: graft-recipient weight ratio, NS: not significant $(\mathrm{P} \geq 0.10)$. 
arrhythmia, transfusion of packed red blood cells, administered drugs (calcium chloride, sodium bicarbonate, or vasopressors), and laboratory data (serum $\mathrm{Na}^{+}$, lactate, and base deficit). MBP and heart rate data were taken at $10,20,30,60,90,120,150$, and 180 min in each phase. The prognosis of patients with ALF who received PMV was determined by assessing: death during the first postoperative month, duration of intensive care unit stay, early graft dysfunction (total bilirubin $>10 \mathrm{mg} / \mathrm{dl}$, prothrombin time $\geq 17 \mathrm{~s}$, encephalopathy grade 4 , within postoperative day 7), acute renal failure (decrease in glomerular filtration rate $>75 \%$, serum creatinine $>4.0 \mathrm{mg} / \mathrm{dl}$ or tripling, urine output below $0.3 \mathrm{ml} / \mathrm{kg} / \mathrm{h}$ for $24 \mathrm{~h}$ ), cardiac complications (uncontrolled arrhythmia, newly developed coronary arterial disease), and infections (surgical site, blood, urinary, pulmonary by culture or radiologic finding) during 1 month after LT as post-transplant variables.

Perioperative variables were compared between patients with and without PMV. Unpaired t-tests or Mann-Whitney U-tests were used to compare intergroup differences, and chi-squared tests were conducted on categorical variables. The data are expressed as means \pm standard deviation, medians (interquartile range), or numbers (percentage) for the univariate analyses. Continuous perioperative variables were dichotomized at the median, quartile, or a clinically meaningful point if necessary. Potentially significant perioperative variables $(\mathrm{P}<0.10)$ identified by the univariate analyses were entered into a forward and backward stepwise multivariate logistic regression analysis to fit a prediction model for PMV after LT for ALF. The sensitivity

Table 2. Comparison of Intraoperative Variables between Patients with and without Prolonged Posttransplant Mechanical Ventilation after Liver Transplantation

\begin{tabular}{|c|c|c|c|}
\hline & \multicolumn{2}{|c|}{ Prolonged posttransplant mechanical ventilation ( $\geq 24 \mathrm{~h}$ ) } & \multirow{2}{*}{$\mathrm{P}$} \\
\hline & No $(\mathrm{n}=71)$ & Yes $(\mathrm{n}=24)$ & \\
\hline Surgical time (min) & $603.8 \pm 129.6$ & $599.6 \pm 133.0$ & NS \\
\hline \multicolumn{4}{|l|}{ Mean blood pressure fluctuation ( $\mathrm{mmHg}$ ) } \\
\hline Preanhepatic phase & $9.7 \pm 4.7$ & $12.0 \pm 5.7$ & 0.051 \\
\hline Anhepatic phase & $6.8 \pm 6.1$ & $6.0 \pm 3.9$ & NS \\
\hline Postanhepatic phase & $6.8 \pm 4.1$ & $5.7 \pm 2.7$ & NS \\
\hline \multicolumn{4}{|l|}{ Heart rate fluctuation (beats/min) } \\
\hline Preanhepatic phase & $7.6 \pm 4.2$ & $8.6 \pm 4.3$ & NS \\
\hline Anhepatic phase & $4.2 \pm 4.4$ & $5.5 \pm 6.2$ & NS \\
\hline Postanhepatic phase & $5.7 \pm 5.8$ & $5.4 \pm 2.9$ & NS \\
\hline Mean PAP (mmHg); initial & $18.8 \pm 4.8$ & $19.9 \pm 6.5$ & NS \\
\hline last & $18.4 \pm 3.2$ & $19.4 \pm 3.7$ & NS \\
\hline CVP $\left(\mathrm{cmH}_{2} \mathrm{O}\right)$; initial & $9.8 \pm 3.6$ & $10.8 \pm 5.6$ & NS \\
\hline last & $9.4 \pm 3.3$ & $9.5 \pm 2.9$ & NS \\
\hline Hourly urine output (ml/kg/h) & $1.54 \pm 0.98$ & $1.09 \pm 0.81$ & 0.024 \\
\hline \multicolumn{4}{|l|}{ Laboratory findings } \\
\hline $\mathrm{Na}^{+}(\mathrm{mEq} / \mathrm{L}) ;$ last & $139.5 \pm 6.4$ & $140.4 \pm 6.1$ & NS \\
\hline intraoperative change & $1.5 \pm 4.9$ & $-0.2 \pm 3.4$ & NS \\
\hline Base deficit $(\mathrm{mEq} / \mathrm{L})$; last & $-5.9 \pm 3.8$ & $-5.0 \pm 4.3$ & NS \\
\hline intraoperative change & $-1.8 \pm 7.8$ & $-0.4 \pm 8.5$ & NS \\
\hline Lactate $(\mathrm{mg} / \mathrm{dl}) ;$ last & $6.4 \pm 3.0$ & $5.7 \pm 3.1$ & NS \\
\hline intraoperative change & $3.2 \pm 2.7$ & $1.6 \pm 2.6$ & NS \\
\hline Oxygen index $\left(\mathrm{PaO}_{2} / \mathrm{FiO}_{2}\right)$; initial & $353.7 \pm 142.7$ & $292.5 \pm 122.6$ & 0.071 \\
\hline last & $374.9 \pm 100.4$ & $324.9 \pm 108.4$ & 0.048 \\
\hline \multicolumn{4}{|l|}{ Blood transfusion } \\
\hline Packed red blood cell & $8.0(5.0-14.0)$ & $9.5(6.0-12.25)$ & NS \\
\hline Fresh frozen plasma & $7.0(4.0-12.0)$ & $9.0(5.25-9.0)$ & NS \\
\hline \multicolumn{4}{|l|}{ Administered drugs } \\
\hline Furosemide (mg) & $30.0(10.0-40.0)$ & $32.5(10.0-80.0)$ & NS \\
\hline Calcium chloride (mg) & $600.0(300.0-1200.0)$ & $975.0(37.5-2537.0)$ & NS \\
\hline Sodium bicarbonate (mg) & $60.0(0.0-110.0)$ & $40.0(0.0-140.0)$ & NS \\
\hline Vasopressors administered & & & NS \\
\hline None & $14(19.7 \%)$ & $3(12.5 \%)$ & \\
\hline Dopamine or dobutamine & $12(16.9 \%)$ & $7(29.2 \%)$ & \\
\hline Epinephrine or norepinephrine & $23(32.4 \%)$ & $9(37.5 \%)$ & \\
\hline Both combined & $22(31.0 \%)$ & $5(20.8 \%)$ & \\
\hline Long vasopressors infusion ( $\geq 3 \mathrm{~h}$ ) & $39(54.9 \%)$ & $16(66.7 \%)$ & NS \\
\hline Arrhythmia & $1(1.4 \%)$ & $2(8.3 \%)$ & 0.094 \\
\hline
\end{tabular}

Data are shown as mean \pm SD or medians (interquartile range) and number (\%). PAP: pulmonary artery pressure, CVP: central venous pressure. 
Table 3. Independent Risk Factors for Prolonged Posttransplant Mechanical Ventilation after Liver Transplantation

\begin{tabular}{|c|c|c|c|c|c|}
\hline & \multirow{2}{*}{$\beta$} & \multirow{2}{*}{ Odds ratio } & \multicolumn{2}{|c|}{$95 \%$ CI } & \multirow{2}{*}{$\mathrm{P}$} \\
\hline & & & Lower & Upper & \\
\hline Severe hepatic encephalopathy (grade III, IV) & 1.570 & 4.82 & 1.22 & 18.9 & 0.025 \\
\hline Mean blood pressure fluctuation during the preanhepatic phase & 1.550 & 4.71 & 1.44 & 15.0 & 0.009 \\
\hline Hourly urine output $(<0.5 \mathrm{ml} / \mathrm{kg} / \mathrm{h})$ & 1.410 & 4.08 & 1.15 & 14.5 & 0.029 \\
\hline Area under receiver operating characteristic curve & 0.757 & & 0.64 & 0.87 & $<0.001$ \\
\hline
\end{tabular}

and unbiased estimate of the model were evaluated using area under the receiver operating characteristic curve analysis. All tests were two-sided, and null hypotheses of no difference were rejected at $\mathrm{P}$ values $<0.05$. The statistical analyses were conducted using the Statistical Package for the Social Sciences version 18.0 (SPSS, Inc., Chicago, IL, USA).

\section{Results}

Of 101 patients with ALF who received LTs during the study period, 95 were eligible for our study. Six cases were excluded because of missing critical variables such as PMV duration. Hepatitis B virus was the most common cause of ALF $(71.6 \%, n$ $=68)$. The other causes were hepatitis A virus $(n=10)$, hepatitis C virus $(n=6)$, alcoholic liver failure $(n=3)$, and drug-induced or unknown hepatitis $(n=8)$. Living donor LTs $(n=79)$ were more frequent than cadaveric donor LTs, and all cases were performed as emergency surgery. Mean duration of PMV was $37.2 \pm 94.5 \mathrm{~h}$ (median, 13.0; range, 10.6-24.0 h). A fourth (n $=24$ ) of the patients received mechanical ventilator support longer than $24 \mathrm{~h}$ after LT, and were categorized as the 'with PMV' group.

Table 1 shows the preoperative variables of patients in the with- and without-PMV after LT groups. No significant intergroup differences were found in age, sex, body mass index, cause of ALF, Child-Pugh-Turcotte class, or past history of other diseases. However, the incidence of factors related to poor physical condition, such as high MELD scores, preoperative intubation, and continuous renal replacement therapy was higher in the with- than in the without-PMV group $(\mathrm{P}<0.05)$. The risk of hepatic encephalopathy more severe than confusion (grade III) in the with-PMV group was approximately four times of that in those in the without-PMV group (33.3\% vs $8.5 \%$, $\mathrm{P}=0.003)$. No intergroup differences were found in donorrelated variables.

Intraoperative MBP was less stable in the with-PMV group and fluctuated during the preanhepatic phase compared with the without-PMV group; however, the difference did not reach statistical significance (Table 2). Patients with PMV had significantly lower hourly urine output throughout all LT phases $(1.09 \pm 0.81$ vs $1.54 \pm 0.98 \mathrm{ml} / \mathrm{kg} / \mathrm{h}, \mathrm{P}=0.024)$ and a lower
Table 4. Comparison of Posttransplant Complications between Patients with and without Prolonged Posttransplantation Mechanical Ventilation after Liver Transplantation

\begin{tabular}{lccc}
\hline & \multicolumn{2}{c}{$\begin{array}{c}\text { Prolonged posttransplant } \\
\text { mechanical ventilation }(\geq 24 \mathrm{~h})\end{array}$} & \multirow{2}{*}{$\mathrm{P}$} \\
\cline { 2 - 3 } & No $(\mathrm{n}=71)$ & Yes $(\mathrm{n}=24)$ & \\
\hline Death & $8(11.3 \%)$ & $7(29.2 \%)$ & 0.038 \\
Stay at intensive care unit (d) & $7.1 \pm 2.7$ & $12.9 \pm 10.4$ & 0.012 \\
Early allograft dysfunction & $9(12.7 \%)$ & $3(12.5 \%)$ & NS \\
Acute renal failure & $12(16.9 \%)$ & $3(12.5 \%)$ & NS \\
Cardiac complication & $3(4.2 \%)$ & $3(12.5 \%)$ & NS \\
Overall infection & $42(59.2 \%)$ & $11(45.8 \%)$ & NS \\
\hline
\end{tabular}

Data are presented as number (\%) and mean \pm SD. NS: statistically not significant $(P \geq 0.05)$.

oxygen index immediately prior to the end of surgery than did those without PMV. No significant intergroup differences were found in blood transfusion, administered drugs including opioids, or vasopressors.

The multivariate analysis revealed three perioperative independent risk factors for PMV after LT for ALF (Table 3). Severe hepatic encephalopathy ( $\geq$ grade III), fluctuation in MBP during the preanhepatic phase, and intraoperative oliguria (hourly urine output $<0.5 \mathrm{ml} / \mathrm{kg} / \mathrm{h}$ ) were associated with a fourfold increase in the risk of PMV after LT $(\mathrm{P}<0.05)$. The area under the receiver operating characteristic curve evaluation indicated that our prediction model for PMV after LT for ALF was adequate and unbiased for clinical use $(\mathrm{P}<0.001)$. In the posttransplant outcome, ALF patients in the with-PMV group had a higher 1-month mortality rate and longer intensive care unit stays compared with those in the without-PMV group ( $\mathrm{P}$ $<0.05$, Table 4). The main cause of death in patients with ALF was multiorgan failure involving graft dysfunction, renal failure, and severe cerebral edema $(71.4 \%, \mathrm{n}=5)$. Other causes of death were acute respiratory distress syndrome $(14.2 \%, \mathrm{n}=1)$, and sepsis $(14.2 \%, \mathrm{n}=1)$.

\section{Discussion}

The development of surgical techniques and immunosup- 
pressive agents has markedly increased the long-term survival rate of LT patients [11]. Nonetheless, a number of transplant patients develop respiratory distress in the intensive care unit after surgery, resulting in adverse post-transplant outcomes [12]. In particular, patients with ALF have a higher risk of long-term perioperative mechanical ventilation support than do patients with other chronic liver diseases. Neurological deterioration accompanied by intracranial hypertension is highly correlated with adverse respiratory manifestations in patients with ALF. Thus, endotracheal intubation and prolonged mechanical ventilation support is not uncommon in these patients, particularly when severe encephalopathy (more severe than confusion) is present [13]. We found that, as expected, the average duration of PMV was significantly longer in patients with ALF $(37.2 \mathrm{~h})$ than in other LT patients (5-24 h) [14]. Moreover, a fourth of the patients in our study required mechanical ventilation support for longer than $24 \mathrm{~h}$.

According to Plevak and Torsher [15], posttransplant management in the $48 \mathrm{~h}$ after LT is the most critical for recipient prognosis. Thus, the primary goal of our study was to evaluate the effect of delayed respiratory recovery immediately after LT on the prognosis of patients with ALF. We found that PMV was associated with longer stays in the intensive care unit and a threefold increase in the 1-month mortality rate, providing strong evidence for the deleterious effects of PMV on the prognosis of ALF LT recipients.

As expected, poor preoperative physical condition was significantly associated with postoperative PMV complications in patients with ALF. In particular, high MELD scores and the use of techniques such as intubation or continuous renal replacement therapy were significantly associated with PMV support. Although preoperative intubation and continuous renal replacement therapy could not be included in the final prediction model for PMV, it is likely that they were associated with the significant predictors for PMV, hepatic encephalopathy and intraoperative urine output. The King's College Criteria are widely used to assess prognosis in patients with ALF; however, the MELD score was developed and validated recently as the most sensitive predictor of survival following LT. However, the predictive accuracy of the MELD score for ALF has not been documented sufficiently. Rutherford et al. [16] compared the MELD score and King's College Criteria with early clinical prognostic indicators such as age $<50$ years, jaundice to encephalopathy interval $>7$ days, grade III or IV hepatic encephalopathy, presence of cerebral edema, prothrombin time $>35 \mathrm{~s}$, and creatinine $>1.5 \mathrm{mg} / \mathrm{dl}$ in a cohort of patients with fulminant hepatic failure. They found that the early clinical prognostic indicators were superior to the MELD and King's College criteria for predicting the clinical outcome of patients with fulminant hepatic failure. Hepatic encephalopathy is among the primary clinical prognostic indicators for patients with ALF.

The precise pathogenesis of hepatic encephalopathy in ALF is not well understood; however, previous studies have suggested an important role for elevated circulatory neurotoxic substances, ammonia in particular. Experimental studies have demonstrated ammonia-induced changes in neurotransmitter synthesis and release, neuronal oxidative stress, impaired mitochondrial function, and osmotic disturbances resulting from astrocytic metabolism of ammonia to glutamine in hepatic encephalopathy. The net results of these changes are marked alterations in cerebral function and astrocyte swelling [17]. The severity of cerebral edema affects the survival rates of patients with ALF and can be assessed clinically by determining the interval from initial jaundice to hepatic encephalopathy [18]. The severity of hepatic encephalopathy must be considered in the prognosis of patients who receive PMV after LT. Biancofiore et al. [19] reported that early posttransplant extubation was hindered by hepatic encephalopathy, intraoperative blood transfusion, and the efficacy of kidney and cardiopulmonary function. In the present study, patients with PMV after LT had a fourfold higher risk of severe hepatic encephalopathy ( $\geq$ grade III, confusion) than those without PMV, suggesting a significant effect of severe encephalopathy on posttransplant outcome.

One goal of the present study was to draw attention to the intraoperative factors associated with post-transplant mechanical ventilation. Stable blood pressure and urine output reflect the intraoperative hemodynamic circulatory state. Several studies have examined intraoperative hemodynamic changes; however, their effect on post-LT outcomes is controversial. Milan et al. [20] reported that intraoperative hemodynamic changes did not have a significant impact on 30-day survival rate or graft quality after surgery. In contrast, Hori et al. [21] found that hemodynamic stability was associated with better graft function, defined by a lower total bilirubin level and shorter intensive care unit stays. In the present study, fluctuating blood pressure during the preanhepatic phase was associated with an increased risk of PMV after transplantation. In contrast to the preanhepatic phase, the anhepatic and postanhepatic phases were not significantly associated with the need for PMV support. During these periods, several factors contributed to hemodynamic instability, such as inferior vena cava clamping and reperfusion of the graft. However, we did not observe sudden, short-term fluctuations in MBP. The lack of detailed data collected at short intervals regarding fluctuations in vital signs may be a limitation of our study; however, our results can be interpreted to suggest that long-term hemodynamic instability has a more significant impact on postoperative outcomes than short-term instability.

Although several devices have been developed to measure beat-to-beat variability in vital signs, such as the PiCCO monitor 
system (PULSION Medical Systems, Munich, Germany), the LiDCO monitoring system (LiDCO Ltd., London, UK), and the uncalibrated FloTrac/Vigileo device (Edwards Life Sciences, Irvine, CA, USA), expressing gross and time-sequential trends in intraoperative fluctuations using a single index is difficult [22]. Thus, we used the standard deviation of MBP or heart rate as the expression of vital sign fluctuation, and tested its usefulness as a clinical predictor of the postoperative outcome. The actual link between MBP fluctuation and mechanical ventilation is not intuitively obvious. However, it is clear that circulatory stability provides a constant blood supply to the brain, lung, liver graft, which is more conducive to physical recovery than fluctuating vital signs. Anesthesiologists have the greatest difficulty managing vital signs during the preanhepatic phase of LT because of the massive blood loss and body fluid turnover due to intensive surgical dissection. Thus, fluctuating blood pressure is common during this phase, which may result in hypovolemia and an urgent need for fluid replacement [23]. Hypovolemia is commonly coupled with hypotension, and causes a deterioration in systemic circulation and a reduction in organ blood flow. Cerebral blood flow autoregulation is often impaired in patients with ALF; the underlying mechanism has not been clearly defined however, it appears to be strongly linked to damaged liver function. Impaired cerebral autoregulation decreases the body's ability to protect the cerebral circulation from systemic circulatory instability. Thus, hypovolemia or arterial hypotension can readily cause brain hypoxia. Cerebral blood flow may increase to disproportionately high levels that exceed metabolic demand, whereas blood pressure remains within the normal physiological range. This can be linked to the subsequent generation of cerebral edema (vasogenic type) [24]. Anesthetic agents administered during LT can further deteriorate cerebral autoregulation in patients with ALF [25]. It is likely that cerebral hypoxia and cerebral edema have a profound effect on the recovery of brain function associated with extubation during the immediate post-transplant period.

Preoperatively, renal impairment is frequently a problem for patients with end-stage liver disease LT recipients. However, renal dysfunction may develop during the immediate post-transplant period [26]. Thus, aggressive protection of the kidneys is an important aspect of intraoperative management in LT recipients to prevent post-transplant renal dysfunction. Intraoperatively, oliguria (reduced hourly urine output $<0.5 \mathrm{ml} / \mathrm{kg} / \mathrm{h}$ ) is a simple and convenient indicator of renal function during surgery and may reveal ineffective management of systemic blood pressure and volume and, ultimately, visceral organ perfusion [27]. Additionally, intraoperative oliguria may impact on the need for PMV in terms of metabolism. Oliguria may decrease the clearance of intraoperative metabolites, fluids, and drugs; thus, their harmful effects on graft, brain, and respiratory functions may be augmented in cases of damaged hepatic clearance. Preexisting renal disease requiring continuous renal replacement therapy may contribute to severe intraoperative oliguria. However, failed efforts by anesthesiologists to protect the kidney during LT may also play a role in the development of intraoperative oliguria. A similar amount of fluid solutions, diuretics, and vasopressors was administered to the with- and without-PMV groups during LT in our study. Thus, a more balanced treatment regime tailored to the patient should be used to prevent aggravation of cerebral edema and intraoperative oliguria in patients with ALF who received PMV.

Hypervolemia has been reported to increase the lung 'wetto-dry ratio' with impairment of oxygenation, and is associated with alveolar and endothelial cell damage. Accordingly, most respiratory care guidelines in intensive care units endorse hypovolemic or normovolemic status to prevent acute lung injury and provide the optimal outcome in mechanically ventilated patients [28]. Contrary to these guidelines, our findings indicate that intraoperative hypovolemia may be harmful to mechanically ventilated ALF LT recipients. It could be argued that the key factor in managing mechanically ventilated patients with ALF is not the condition of the lungs, but rather the neurological recovery of the recipient. Neurological recovery in ALF transplant recipients may rely on the functional recovery of the grafted liver to metabolize or remove toxic materials from the brain. In general, most preoperative pulmonary complications in LT recipients originate from massive ascites, which is slowly relieved following surgical opening of the abdominal wall during LT. Thus, pulmonary complications do not have a significant effect on posttransplant ventilation [29]. In the present study, the final oxygen indices measured during LT were slightly higher in recipients with PMV than in those without. However, the mean oxygen indices of the patients with PMV remained within the normal range, and none of the pulmonary variables were identified as independent risk factors in the predictive model for PMV. These results suggest that, similar to recipients of LT for other conditions, pulmonary complications do not play a significant role in the outcome of PMV in patients with ALF [30].

The present study has several limitations owing to the retrospective design. First, deletion of perioperative variables with critical missing data could not be avoided. Although we examined the main factors associated with mechanical ventilation, inclusion of all known predictors of PMV would have resulted in an excessive number of variables. Second, the donation source was not homogeneous. Although no difference in the necessity for in PMV was found between living and cadaveric donors, the type of donor could not be restricted because of the small number of LT performed for ALF. Third, this was not a multiple center-based study, and the LTs were performed by two 
surgeons. Fourth, the measurement of MBP fluctuations was limited during the anhepatic and neohepatic phases as a result of the data collection interval, as discussed previously. Finally, the mechanism underlying the relationship between PMV and the intraoperative circulatory status could not be definitively clarified. However, clarification of the intraoperative factors associated with PMV in patients with ALF is a novel and meaningful aspect of the present study. To prevent the need for PMV in patients with ALF, closer monitoring of urine output and increased efforts to prevent oliguria and blood pressure fluctuations using fluid solutions, diuretics, and vasopressors may be required of anesthesiologists working in LT field.

In conclusion, we found that PMV after LT for ALF is closely related to deleterious posttransplant outcomes. In addition to known risk factors, hepatic encephalopathy, fluctuations in intraoperative blood pressure, and oliguria were identified as independent predictors of PMV after LT in patients with ALF. Attention to the hemodynamic state and more aggressive efforts to improve the circulatory condition are required to avoid PMV and optimize the post-transplant prognosis in patients with ALF.

\section{References}

1. Lo CM. Living donor liver transplantation for acute liver failure: no other choice. Liver Transpl 2012; 18: 1005-6.

2. O'Grady J. Liver transplantation for acute liver failure. Best Pract Res Clin Gastroenterol 2012; 26: 27-33.

3. Lim YS. Acute liver failure in Korea: etiology, prognosis and treatment. Korean J Hepatol 2010; 16: 5-18.

4. Castaldo ET, Chari RS. Liver transplantation for acute hepatic failure. HPB (Oxford) 2006; 8: 29-34.

5. Wong DT, Cheng DC, Kustra R, Tibshirani R, Karski J, Carroll-Munro J, et al. Risk factors of delayed extubation, prolonged length of stay in the intensive care unit, and mortality in patients undergoing coronary artery bypass graft with fast-track cardiac anesthesia: a new cardiac risk score. Anesthesiology 1999; 91: 936-44.

6. Neelakanta G, Sopher M, Chan S, Pregler J, Steadman R, Braunfeld M, et al. Early tracheal extubation after liver transplantation. J Cardiothorac Vasc Anesth 1997; 11: 165-7.

7. Ferraz-Neto BH, Silva ED, Afonso RC, Gregory FH, Goehler F, Meira-Filho SP, et al. Early extubation in liver transplantation. Transplant Proc 1999; 31: 3067-8.

8. Polson J, Lee WM. AASLD position paper: the management of acute liver failure. Hepatology 2005; 41: 1179-97.

9. MacIntyre NR, Cook DJ, Ely EW Jr, Epstein SK, Fink JB, Heffner JE, et al. Evidence-based guidelines for weaning and discontinuing ventilatory support: a collective task force facilitated by the American College of Chest Physicians; the American Association for Respiratory Care; and the American College of Critical Care Medicine. Chest 2001; 120(6 Suppl): S375-95.

10. Glanemann M, Langrehr J, Kaisers U, Schenk R, Muller A, Stange B, et al. Postoperative tracheal extubation after orthotopic liver transplantation. Acta Anaesthesiol Scand 2001; 45: 333-9.

11. Schrem H, Till N, Becker T, Bektas H, Manns MP, Strassburg CP, et al. Long-term results after liver transplantation. Chirurg 2008; 79: 1219.

12. Lee JM, Chang HW, Park CS, Park HJ, Kim JE, Choi JH. Evaluation of mechanical ventilation and its influencing factors between the living related liver transplantation and cadaveric whole liver transplantation. Korean J Anesthesiol 2005; 49: 816-21.

13. Steadman RH, Van Rensburg A, Kramer DJ. Transplantation for acute liver failure: perioperative management. Curr Opin Organ Transplant 2010; $15: 368-73$.

14. Choi JH, Kim TH, Lee JM. Evaluation of usefulness of perioperative risk factors which affect early or delayed extubation after liver transplantation. Korean J Anesthesiol 2003; 44: 847-52.

15. Plevak DJ, Torsher LC. Fast tracking in liver transplantation. Liver Transpl Surg 1997; 3: 447-8.

16. Rutherford A, King LY, Hynan LS, Vedvyas C, Lin W, Lee WM, et al. Development of an accurate index for predicting outcomes of patients with acute liver failure. Gastroenterology 2012; 143: 1237-43.

17. Bernal W, Auzinger G, Sizer E, Wendon J. Intensive care management of acute liver failure. Semin Liver Dis 2008; 28: 188-200.

18. O'Grady JG, Schalm SW, Williams R. Acute liver failure: redefining the syndromes. Lancet 1993; 342: 273-5.

19. Biancofiore G, Romanelli AM, Bindi ML, Consani G, Boldrini A, Battistini M, et al. Very early tracheal extubation without predetermined criteria in a liver transplant recipient population. Liver Transpl 2001; 7: 777-82.

20. Milan Z, Taylor C, Duncan B, Kedilaya H, Sylvester D. Statistical modeling of hemodynamic changes during orthotopic liver transplantation: predictive value for outcome and effect of marginal donors. Transplant Proc 2011; 43: 1711-5.

21. Hori T, Yagi S, Iida T, Taniguchi K, Yamagiwa K, Yamamoto C, et al. Optimal systemic hemodynamic stability for successful clinical outcomes after adult living-donor liver transplantation: prospective observational study. J Gastroenterol Hepatol 2008; 23: e170-8.

22. Porhomayon J, Zadeii G, Congello S, Nader ND. Applications of minimally invasive cardiac output monitors. Int J Emerg Med $2012 ; 5: 18$.

23. Bliacheriene F, Machado SB, Fonseca EB, Otsuke D, Auler JO Jr, Michard F. Pulse pressure variation as a tool to detect hypovolaemia during pneumoperitoneum. Acta Anaesthesiol Scand 2007; 51: 1268-72. 
24. Ardizzone G, Arrigo A, Panaro F, Ornis S, Colombi R, Distefano S, et al. Cerebral hemodynamic and metabolic changes in patients with fulminant hepatic failure during liver transplantation. Transplant Proc 2004; 36: 3060-4.

25. Zheng Y, Villamayor AJ, Merritt W, Pustavoitau A, Latif A, Bhambhani R, et al. Continuous cerebral blood flow autoregulation monitoring in patients undergoing liver transplantation. Neurocrit Care 2012; 17: 77-84.

26. Tinti F, Umbro I, Giannelli V, Merli M, Ginanni Corradini S, Rossi M, et al. Acute renal failure in liver transplant recipients: role of pretransplantation renal function and 1-year follow-up. Transplant Proc 2011; 43: 1136-8.

27. Shamir MY, Kaplan L, Marans RS, Willner D, Klein Y. Urine flow is a novel hemodynamic monitoring tool for the detection of hypovolemia. Anesth Analg 2011; 112: 593-6.

28. Silva PL, Cruz FF, Fujisaki LC, Oliveira GP, Samary CS, Ornellas DS, et al. Hypervolemia induces and potentiates lung damage after recruitment maneuver in a model of sepsis-induced acute lung injury. Crit Care 2010; 14: R114.

29. Vater Y, Dembo G, Martay K, Vitin A, Amar E, Weinbroum AA. Ascites characterizes perioperative clinical indices better than preoperative body mass index. A study in orthotopic liver transplant candidates. Minerva Anesthesiol 2012; 78: 910-9.

30. Serste T, Moreno C, Francoz C, Razek WA, Paugham C, Belghitti J, et al. The impact of preoperative hepatic hydrothorax on the outcome of adult liver transplantation. Eur J Gastroenterol Hepatol 2010; 22: 207-12. 